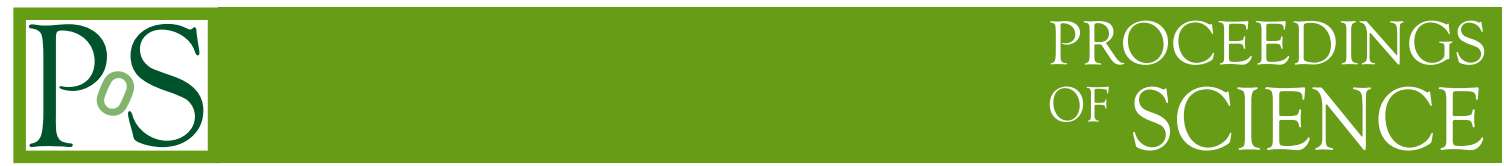

\title{
On the decoupling of mirror fermions
}

\author{
Joel Giedt* \\ Department of Physics, Applied Physics, and Astronomy, \\ Rensselaer Polytechnic Institute, 110 8th St., Troy, New York, 12180, USA \\ E-mail: giedt jerpi.edu
}

\section{Chen Chen}

\section{Erich Poppitz}

Department of Physics, University of Toronto, Toronto, ON M5S 1A7, Canada

E-mail: poppitz@physics.utoronto.ca

We study an approach to chiral gauge theories on the lattice that involves decoupling "mirror" fermions from a vector-like theory. We have computed the polarization tensor in the "3-4-5" theory and find a directional discontinuity that appears to be nonzero in the continuum limit. This strongly suggests that the mirror fermions do not decouple.

31st International Symposium on Lattice Field Theory - LATTICE 2013

July 29 - August 3, 2013

Mainz, Germany

${ }^{*}$ Speaker. 


\section{Introduction}

Strongly-coupled chiral gauge theories are prominent in ideas about what lies beyond the Standard Model. Most attractive channel arguments suggest that they may lead to tumbling dynamics [1], explaining a hierarchy of scales in extended technicolor. In supersymmetric models, strong chiral dynamics is often a key component in models of dynamical supersymmetry breaking (see, e.g., the review [2] for extensive references). For these and other more theoretical reasons, we would like to study strongly-coupled chiral gauge theories from first principles, using a lattice formulation. In contrast to vector-like theories, severe difficulties arise when we try to formulate chiral gauge theories on the lattice. In older approaches, solving the fermion doubling problem resulted in actions that explicitly violated chiral symmetry $[3,4,5]$; e.g., Wilson's fermion discretization [6, 7]. More recently, discretizations that satisfy the Ginsparg-Wilson relation [8] have been developed [9, 10, 11, 12], maintaining lattice chiral symmetry [13]. In spite of this progress, problems surfaced with this approach as it applies to chiral gauge theories. For instance, for overlap fermions the fermion measure had a gauge-background dependent phase.

Various attempts have been made to overcome the problems. For instance, the recent approach of [14] involves non-perturbative gauge fixing. By contrast, Lüscher's approach defines the phase of the fermion determinant through consistency conditions (see the review [15]). In the case of Abelian chiral gauge theories, the phase can be determined for arbitrary gauge backgrounds though to date no numerical implementation of this has been performed. The approach studied in this paper would avoid having to solve Lüscher's consistency conditions. This "mirror fermion decoupling" has been reviewed in [16].

In this approach a vector-like theory is decomposed into "light" particles of one chirality and "mirror" particles of the opposite chirality. Through a strong Yukawa coupling of the mirror fermions to a unitary "Higgs" field it is hoped that they will acquire a mass of order the ultraviolet cutoff. Note that this occurs in the symmetric phase of the Higgs theory, i.e., there is no spontaneous gauge symmetry breaking in our model. The large mass is purely a dynamical effect of the strong Yukawa coupling. This is similar to [17], which used four-fermion interactions instead of a Higgs field.

Earlier efforts $[17,18]$ did not have a clean separation of chiral components of the vector-like theory. Thus both the light and mirror sectors experienced the the strong interactions. The result was that the spectrum did not separate according to the chiral goals. This situation has changed because of the Neuberger-Dirac overlap operator and exact lattice chirality. Using this, there was a proposal to lift the mirror sector with a strong Yukawa coupling that does not affect the light sector [19] (note also the earlier proposal of Ref. [20] using domain wall fermions).

An initial numerical study of this proposal was performed in Ref. [21], using as example the two-dimensional Schwinger model, split into chiral "light" and "mirror" sectors, with strong "mirror" couplings to a unitary scalar in order to, hopefully, lift the mirror sector. Correlation functions of the obvious operators did not show the presence of massless modes in the mirror sector. However, because the mirror spectrum is was anomalous, 't Hooft anomaly matching implied that they ought to be present. The follow-up work [22] employed the polarization tensor, finding a directional discontinuity at zero momentum, a clear indication of a massless state.

Therefore, it is of interest to study a theory where 't Hooft anomaly matching does not imply 


\begin{tabular}{|c|c|c|}
\hline Light Field & Mirror Field & $\mathrm{Q}$ \\
\hline$A_{+}$ & $A_{-}$ & 3 \\
$B_{+}$ & $B_{-}$ & 4 \\
$C_{-}$ & $C_{+}$ & 5 \\
$X_{-}$ & $X_{+}$ & 0 \\
- & $\phi$ & -1 \\
\hline
\end{tabular}

Table 1: Summary of the field content in the 3-4-5 model.

massless modes in the mirror sector. Our recent work considered just such a case [23], and we summarize our results in this proceedings report. It will be seen that the massless modes persist.

\section{The 3-4-5 model}

The "3-4-5 model" is a two-dimensional lattice gauge theory with U(1) gauge invariance. It has three Weyl fermion fields, which we denote $A_{+}, B_{+}, C_{-}$, with charge and chirality $3_{+}, 4_{+}$, and $5-$ respectively. In addition, there is a mirror sector, $3_{-}, 4_{-}$, and $5_{+}$, and we call the respective fields $A_{-}, B_{-}$, and $C_{+}$. In order to construct all of the Yukawa couplings that are needed, a neutral spectator fermion $X_{-}$, with charge and chirality $0_{-}$, is also introduced, together with its mirror sector partner $X_{+}$, with charge and chirality $0_{+}$. Finally, in order to lift the mirror sector, a unitary "Higgs" field $\phi$ with charge -1 is introduced. The 3-4-5 model fields are given in Table 1 .

The dynamics of the gauge field is not supposed to be involved in the mechanism that lifts the mirror fermions, so in our analysis we neglected the gauge field fluctuations and treated it only as a background field. The action of the " $3-4-5$ " model is

$$
\begin{aligned}
S= & S_{\text {light }}+S_{\text {mirror }} \\
S_{\text {light }}= & -\left(\bar{A}_{+} \cdot D_{3} \cdot A_{+}\right)-\left(\bar{B}_{+} \cdot D_{4} \cdot B_{+}\right)-\left(\bar{C}_{-} \cdot D_{5} \cdot C_{-}\right)-\left(\bar{X}_{-} \cdot D_{0} \cdot X_{-}\right) \\
S_{\text {mirror }}= & S_{\kappa}-\left(\bar{A}_{-} \cdot D_{3} \cdot A_{-}\right)-\left(\bar{B}_{-} \cdot D_{4} \cdot B_{-}\right)-\left(\bar{C}_{+} \cdot D_{5} \cdot C_{+}\right)-\left(\bar{X}_{+} \cdot D_{0} \cdot X_{+}\right) \\
& +S_{\text {Yuk.,Dirac }}+S_{\text {Yuk.,Maj }},
\end{aligned}
$$

Here, $D_{q}$ is Neuberger's overlap Dirac operator [12], with charge $q$ on the gauge field. Chiral projections are based on the $\gamma_{5}$ and $\hat{\gamma}_{5}$ operators, as is typical in the overlap formalism. The Yukawa interactions of the mirror sector are

$$
\begin{aligned}
S_{\text {Yuk.,Dirac }}= & y_{30} \bar{A}_{-} X_{+} \phi^{-3}+y_{40} \bar{B}_{-} X_{+} \phi^{-4}+y_{35} \bar{A}_{-} C_{+} \phi^{2}+y_{45} \bar{B}_{-} C_{+} \phi \\
& +y_{30} \bar{X}_{+} A_{-} \phi^{3}+y_{40} \bar{X}_{+} B_{-} \phi^{4}+y_{35} \bar{C}_{+} A_{-} \phi^{-2}+y_{45} \bar{C}_{+} B_{-} \phi^{-1} \\
S_{\text {Yuk.,Maj. }}= & h_{30} A_{-}^{T} \gamma_{2} X_{+} \phi^{3}+h_{40} B_{-}^{T} \gamma_{2} X_{+} \phi^{4}+h_{35} A_{-}^{T} \gamma_{2} C_{+} \phi^{8}+h_{45} B_{-}^{T} \gamma_{2} C_{+} \phi^{9} \\
& -h_{30} \bar{X}_{+} \gamma_{2} \bar{A}_{-}^{T} \phi^{-3}-h_{40} \bar{X}_{+} \gamma_{2} \bar{B}_{-}^{T} \phi^{-4}-h_{35} \bar{C}_{+} \gamma_{2} \bar{A}_{-}^{T} \phi^{-8} \\
& -h_{45} \bar{C}_{+} \gamma_{2} \bar{B}_{-}^{T} \phi^{-9} .
\end{aligned}
$$

Each of the terms in (2.2) has an implicit sum over lattice sites: $\bar{A}_{-} X_{+} \phi^{-3}=\sum_{x} \bar{A}_{-, x} X_{+, x} \phi_{x}^{-3}$. Finally, the field $\phi_{x}=e^{i \eta_{x}},|\eta| \leq \pi$, is a unitary Higgs field of charge -1 with a kinetic term:

$$
S_{\kappa}=\frac{\kappa}{2} \sum_{x} \sum_{\mu}\left[2-\left(\phi_{x}^{*} U_{\mu}^{*}(x) \phi_{x+\hat{\mu}}+h . c .\right)\right] .
$$




\begin{tabular}{cccccccc}
\hline$h_{30}$ & $h_{40}$ & $h_{35}$ & $h_{45}$ & $y_{30}$ & $y_{40}$ & $y_{35}$ & $y_{45}$ \\
\hline 30.3214 & 3.08123 & 3.00278 & 23.7109 & 1.0 & 1.0 & 1.0 & 1.0 \\
\hline
\end{tabular}

Table 2: A set of Yukawa coupling constants that we found by optimizing narrowness of the complex phase distribution for an $8 \times 8$ lattice.

\section{Preliminaries of the calculation}

\subsection{The mirror polarization tensor}

The vacuum polarization tensor is defined as:

$$
\left.\Pi_{\mu v}(x, y) \equiv \frac{\delta^{2} \ln Z[A]}{\delta A(x) \delta A(y)}\right|_{A=0} .
$$

Just as the partition function splits into the product of "mirror" and "light" parts, so does the polarization operator:

$$
\Pi_{\mu \nu}(x, y)=\Pi_{\mu \nu}^{\text {light }}(x, y)+\Pi_{\mu \nu}^{\text {mirror }}(x, y),
$$

\subsection{Complex phase}

The fermion determinant of our theory is not real. To overcome this feature, we have studied the dependence of the phase distribution on the values of the Yukawa coupling parameters. We have optimized these parameters in order to have a narrow phase distribution, leading to the choice of couplings in Table 2. Since we calculate the fermion determinant at each sampling step of our simulation, we include the complex phase in our expectation values.

\subsection{Probing for massless particles}

In the continuum, the contribution to the Fourier transform of the real part of the polarization tensors due to massless particles takes the form:

$$
\tilde{\Pi}_{\mu v}(k)=2 C \frac{\delta_{\mu v} k^{2}-k_{\mu} k_{v}}{k^{2}} .
$$

A single free charge- $q$ Weyl fermion, gives a contribution equal to that of a half charge- $q$ Dirac fermion: $2 C_{\text {fermion }} \simeq-\frac{1}{2 \pi} q^{2}$. The way to look for a massless pole in (3.3) is to notice that (3.3) has a directional limit as $k \rightarrow 0$ :

$$
\left.\tilde{\Pi}_{11}(\phi)\right|_{k \rightarrow 0}=C(1-\cos 2 \phi),\left.\quad \tilde{\Pi}_{21}(\phi)\right|_{k \rightarrow 0}=-C \sin 2 \phi,
$$

where $\phi$ is the angle of approach to origin measured from the positive- $k_{1}$ axis. Therefore if there is a massless particle in the spectrum of the mirror theory, we would expect $\tilde{\Pi}_{\mu \nu}^{\text {mirror, }}$ has the following behavior as $k \rightarrow 0$ :

$$
\begin{aligned}
\tilde{\Pi}_{11}\left(45^{o}\right) & =-\tilde{\Pi}_{21}\left(45^{o}\right)=C, \quad \tilde{\Pi}_{11}\left(90^{o}\right)=2 C, \\
\tilde{\Pi}_{11}\left(0^{o}\right) & =\tilde{\Pi}_{21}\left(0^{o}\right)=\tilde{\Pi}_{21}\left(90^{o}\right) .
\end{aligned}
$$

On the other hand, if there is no massless particle, we would expect: $\tilde{\Pi}_{\mu v} \sim\left(\delta_{\mu v} k^{2}-k_{\mu} k_{v}\right) / m^{2}$, as $k \rightarrow 0$. Therefore if the mirror sector acquires the desired mass scale, the directional limit behavior (3.4) disappears. 


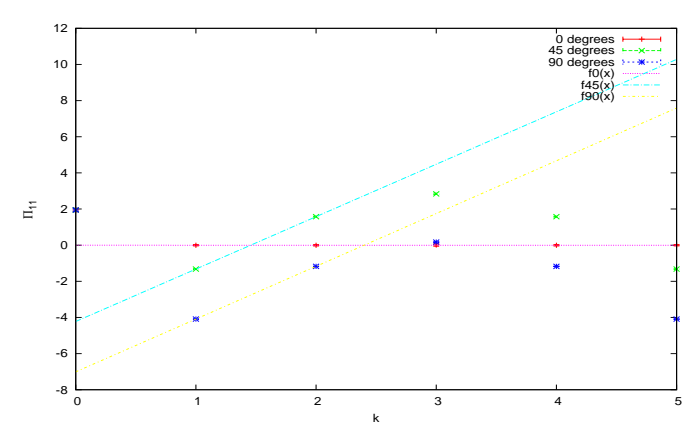

\begin{tabular}{cccc}
\hline$\phi$ & $A(6 \times 6)$ & $A(8 \times 8)$ & $A(10 \times 10)$ \\
\hline $0^{o}$ & $-2.36 \times 10^{-3}$ & $-0.16423(82)$ & $-(0)$ \\
$45^{\circ}$ & -4.22 & $-3.820(98)$ & $-3.764(41)$ \\
$90^{\circ}$ & -7.01 & $-6.43(16)$ & $-6.501(47)$ \\
\hline
\end{tabular}

\section{Results}

For the set of couplings given in Table 2, we computed $\tilde{\Pi}_{\mu \nu}^{\text {mirror, }}(k)$ for $6 \times 6,8 \times 8$ and $10 \times 10$ lattices. The reason for using such small lattices is because the calculation of $\tilde{\Pi}_{\mu \nu}^{\text {mirror, },}(k)$ is rather demanding and scales badly ( $\sim N^{10}$ after having taken into account momentum conservation) with the number of lattice sites $N$ in each direction. We studied three directions in momentum space: $0^{\circ}$ with $\mathbf{k}=(k, 0) ; 45^{\circ}$ with $\mathbf{k}=(k, k) ; 90^{\circ}$ with $\mathbf{k}=(0, k)$. Here $k$ was taken from $0,1, \ldots, N-1$ on an $N \times N$ lattice. The corresponding momenta in physical units are $p=2 \pi k / N a$.

Figure 1 shows the results obtained on the $6 \times 6$ lattice. It has the pattern suggested by (3.4) with $C \approx 4 \approx 50 /(4 \pi)$. In fact, it is remarkably similar to Figs. $2-5$ of Ref. [22], except that the discontinuity is about 50 times larger, consistent with contributions from the three charges that are present in the underlying theory: $3^{2}+4^{2}+5^{2}=50$. The $k \rightarrow 0$ extrapolations are given in the second column of Table 3, where we used a linear fit to the two smallest nonzero $k$ values.

Our results for the $8 \times 8$ and $10 \times 10$ lattices with the same Yukawa couplings are similar to the $6 \times 6$ case, are shown in [23], and have $k \rightarrow 0$ extrapolations given in the other columns of Table 3 . As one can see, the discontinuity constant $C$ is approximately the same as was found on the $6 \times 6$ lattice.

If the physical volume $L \times L$ is held fixed, where $L=N a$, while the number of lattice sites $N \times N$ is increased, this corresponds to decreasing the lattice spacing. The discretization error of the overlap fermions which we are using is known to be $\mathscr{O}\left(a^{2}\right)$. Thus continuum extrapolation obeys

$$
C=b+c(a / L)^{2}+\mathscr{O}\left((a / L)^{4}\right)=b+c N^{-2}+\mathscr{O}\left(N^{-4}\right)
$$

Here, we use the values of $C$ from Table 3 for each of the three values of $N=L / a$ that we have computed. The errors in the value of $C$ are dominated by the difference between the value obtained 


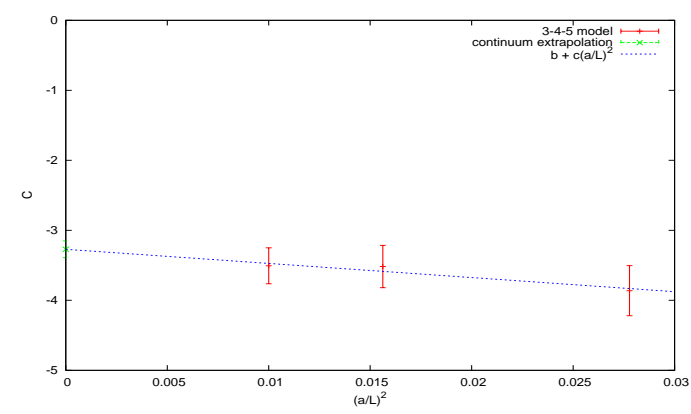

Figure 2: The small- $k$ discontinuity in $\tilde{\Pi}_{11}$ fitted to a linear function of $(a / L)^{2}$. It is seen that the discontinuity appears to approach a nonzero value as $a \rightarrow 0$.

from $45^{\circ}$ versus $90^{\circ}$. Taking this into account, we obtain the fit shown in Figure 2. It can be seen that the linear function in $(a / L)^{2}$ describes the data very well. Furthermore, the linear extrapolation intersects the $a=0$ limit at a nonzero discontinuity, $b=-3.27(12)$. This suggests that in the continuum limit of the 3-4-5 model, the polarization tensor has a directional discontinuity, consistent with having massless modes.

\section{Conclusions}

We have studied the polarization operator of the 3-4-5 mirror theory in order to probe for the existence of massless mirror states. We find evidence that the polarization tensor has a directional discontinuity at $k=0$. This supports the conclusion that the mirror sector fermions do not decouple, but remain massless, in spite of the strong Yukawa dynamics. It is strange that the strong Yukawa interaction does not give mass to the mirror sector, given that 't Hooft anomaly matching does not imply any massless modes in this anomaly free theory. One goal of future work is to understand what the mechanism is for giving massless modes in the mirror sector in this lattice theory.

\section{Acknowledgments}

C.C. and J.G. were supported in part by the Department of Energy, Office of Science, Office of High Energy Physics, Grant No. DE-FG02-08ER41575. E.P. was supported in part by the National Science and Engineering Council of Canada (NSERC). We gratefully acknowledge the use of USQCD computing resources at Fermi National Laboratory, under a Class C allocation.

\section{References}

[1] S. Raby, S. Dimopoulos, and L. Susskind, Tumbling Gauge Theories, Nucl.Phys. B169 (1980) 373.

[2] E. Poppitz and S. P. Trivedi, Dynamical supersymmetry breaking, Ann.Rev.Nucl.Part.Sci. 48 (1998) 307-350, [hep-th/9803107].

[3] H. B. Nielsen and M. Ninomiya, Absence of Neutrinos on a Lattice. 1. Proof by Homotopy Theory, Nucl.Phys. B185 (1981) 20.

[4] H. B. Nielsen and M. Ninomiya, No Go Theorem for Regularizing Chiral Fermions, Phys.Lett. B105 (1981) 219. 
[5] H. B. Nielsen and M. Ninomiya, Absence of Neutrinos on a Lattice. 2. Intuitive Topological Proof, Nucl.Phys. B193 (1981) 173.

[6] L. H. Karsten and J. Smit, Lattice Fermions: Species Doubling, Chiral Invariance, and the Triangle Anomaly, Nucl.Phys. B183 (1981) 103.

[7] L. H. Karsten, Lattice fermions in Euclieadn space-time, Phys.Lett. B104 (1981) 315.

[8] P. H. Ginsparg and K. G. Wilson, A Remnant of Chiral Symmetry on the Lattice, Phys.Rev. D25 (1982) 2649.

[9] D. B. Kaplan, A Method for simulating chiral fermions on the lattice, Phys.Lett. $\mathbf{B 2 8 8}$ (1992) 342-347, [hep-lat/9206013].

[10] R. Narayanan and H. Neuberger, Chiral fermions on the lattice, Phys.Rev.Lett. 71 (1993) 3251-3254, [hep-lat/9308011].

[11] R. Narayanan and H. Neuberger, A Construction of lattice chiral gauge theories, Nucl.Phys. B443 (1995) 305-385, [hep-th/9411108].

[12] H. Neuberger, Exactly massless quarks on the lattice, Phys.Lett. B417 (1998) 141-144, [hep-lat/9707022].

[13] M. Luscher, Exact chiral symmetry on the lattice and the Ginsparg-Wilson relation, Phys.Lett. B428 (1998) 342-345, [hep-lat/9802011].

[14] M. Golterman and Y. Shamir, SU(N) chiral gauge theories on the lattice, Phys.Rev. D70 (2004) 094506, [hep-lat/0404011].

[15] M. Luscher, Chiral gauge theories revisited, hep-th/0102028.

[16] E. Poppitz and Y. Shang, Chiral Lattice Gauge Theories Via Mirror-Fermion Decoupling: A Mission (im)Possible?, Int.J.Mod.Phys. A25 (2010) 2761-2813, [arXiv:1003.5896].

[17] E. Eichten and J. Preskill, Chiral Gauge Theories on the Lattice, Nucl.Phys. B268 (1986) 179.

[18] J. Smit, Fermions on a Lattice, Acta Phys.Polon. B17 (1986) 531.

[19] T. Bhattacharya, M. R. Martin, and E. Poppitz, Chiral lattice gauge theories from warped domain walls and Ginsparg-Wilson fermions, Phys.Rev. D74 (2006) 085028, [hep-lat/ 0605003 ].

[20] M. Creutz, M. Tytgat, C. Rebbi, and S.-S. Xue, Lattice formulation of the standard model, Phys.Lett. B402 (1997) 341-345, [hep-lat/9612017].

[21] J. Giedt and E. Poppitz, Chiral Lattice Gauge Theories and The Strong Coupling Dynamics of a Yukawa-Higgs Model with Ginsparg-Wilson Fermions, JHEP 0710 (2007) 076, [hep-lat/0701004].

[22] E. Poppitz and Y. Shang, Lattice chirality, anomaly matching, and more on the (non)decoupling of mirror fermions, JHEP 0903 (2009) 103, [arXiv: 0901 . 3402].

[23] C. Chen, J. Giedt, and E. Poppitz, On the decoupling of mirror fermions, JHEP 1304 (2013) 131, [arXiv:1211.6947]. 